\title{
Inhibition of TRIM32 Attenuates the Apoptosis, Oxidative Stress and Inflammatory Injury of Podocytes Induced by High Glucose by Affecting the Akt/GSK-3ß/Nrf2 Pathway
}

\section{Zhao Chen}

The Second Affiliated Hospital of Xi'an Jiaotong University

\section{Lifang Tian}

The Second Affiliated Hospital of Xi'an Jiaotong University

\section{Li Wang}

The Second Affiliated Hospital of Xi'an Jiaotong University

\section{Xiaotao Ma}

The Second Affiliated Hospital of Xi'an Jiaotong University

\section{Fuqian Lei}

The Second Affiliated Hospital of Xi'an Jiaotong University

\section{Xianghui Chen}

The First Affiliated Hospital of Xi'an Jiaotong University

Rongguo Fu ( $\nabla$ fu_rongguo@163.com )

The Second Affiliated Hospital of Xi'an Jiaotong University https://orcid.org/0000-0002-5308-9980

\section{Research Article}

Keywords: Akt, diabetic nephropathy, high glucose, podocyte, TRIM32

Posted Date: June 1st, 2021

DOI: https://doi.org/10.21203/rs.3.rs-551617/v1

License: (c) (1) This work is licensed under a Creative Commons Attribution 4.0 International License. Read Full License 


\section{Abstract}

Hyperglycemia-induced oxidative stress of podocytes exerts a major role in the pathological process of diabetic nephropathy. Tripartite motif-containing protein 32 (TRIM32) has been reported as a key protein in the modulation of cellular apoptosis and oxidative stress under various pathological processes. However, whether TRIM32 participates in the regulation of high glucose (HG)-induced injury in podocytes has not been investigated. The aims of this work were to assess the possible role of TRIM32 in mediating HG-induced apoptosis, oxidative stress and inflammatory response in podocytes in vitro. Herein, our results showed a marked increase in TRIM32 expression in HG-exposed podocytes. Loss-of-function experiments showed that the knockdown of TRIM32 improved the viability of HG-stimulated podocytes, and suppressed HG-induced apoptosis, oxidative stress and inflammatory response in podocytes. Further investigation revealed that the inhibition of TRIM32 enhanced the activation of nuclear factor erythroid 2related factor 2 (Nrf2) signaling associated with modulation of the Akt/glycogen synthase kinase-3 $\beta$ (GSK-3 $\beta$ ) axis in podocytes following HG exposure. However, the suppression of Akt abrogated the TRIM32-knockdown-mediated activation of Nrf2 in HG-exposed podocytes. In addition, the knockdown of Nrf2 markedly abolished the TRIM32-inhibition-induced protective effects in HG-exposed podocytes. In summary, the results of this work show that the inhibition of TRIM32 protects podocytes from HGinduced injury by potentiating Nrf2 signaling via the modulation of Akt/GSK-3 $\beta$ signaling. This study indicates a potential role of TRIM32 in mediating podocyte injury during the progression of diabetic nephropathy.

\section{Introduction}

Diabetic nephropathy is a severe complication of diabetes and represents a major contributor to endstage renal disease [1]. Diabetic nephropathy is characterized by glomerular injury and accompanied by proteinuria [2]. Unfortunately, up to $40 \%$ of diabetic patients will eventually develop diabetic nephropathy [3]. However, the treatment of diabetic nephropathy remains a challenge in current years. Glomerular podocytes, major components of the glomerular filtration barrier, plays a vital role in the progression of diabetic nephropathy [4]. Persistent exposure to hyperglycemia evokes podocyte injury via the induction of apoptosis, oxidative stress, and inflammatory response, which is related to the pathogenesis of diabetic nephropathy [5-7]. However, the molecular mechanisms underlying hyperglycemia-evoked podocyte injury remain elusive. Therefore, a better understanding of the molecular mechanisms responsible for hyperglycemia-evoked podocyte injury may offer a new opportunity for the exploitation of innovative therapeutic options for diabetic nephropathy.

Tripartite motif-containing protein 32 (TRIM32) is a member of the TRIM family, which is related to a number of physiological and pathological processes [8]. TRIM32 harbors the typical RING domain, conferring E3 ligase activity, and is capable of ubiquitinating various substrates [9-11]. TRIM32 is widely expressed in adult tissues, and plays a multi-faceted role in diverse processes [12]. The dysregulation of TRIM32 is implicated in numerous pathological conditions, including myopathy, cardiomyopathy, neurological disorder and cancers [13-20]. Moreover, TRIM32 participates in the regulation of diverse 
cellular activities, including differentiation, proliferation, apoptosis, oxidative stress and inflammatory response [21-24].

Nuclear factor erythroid 2-related factor 2 (Nrf2) plays an essential role in organizing the cellular protection network under adverse stimulus [25]. Nrf2 translocates to the nucleus where it is capable of binding to an antioxidant-response-element (ARE) in gene promoters to induce the expression of cytoprotective target genes [26]. Nrf2 is involved in plenty of pathological conditions by affecting apoptosis, oxidative stress and inflammatory responses [27-30]. Notably, increasing evidence has shown that Nrf2 exerts a key role in the pathogenesis of diabetic nephropathy [31-33]. The activation of Nrf2 ameliorates high glucose (HG)-induced apoptosis, oxidative stress and the inflammation of podocytes, which is conducive to curing diabetic nephropathy [34-36]. The activation of Nrf2 is modulated via

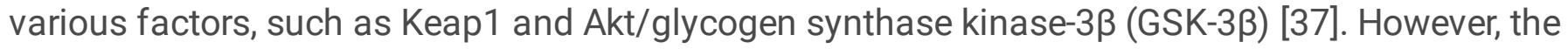
regulation of Nrf2 activation in diabetic nephropathy remains poorly understood.

To date, whether TRIM32 plays a role in diabetic nephropathy is unknown. In this study, we aimed to elucidate the potential role of TRIM32 in regulating podocyte injury induced by HG. Our results showed marked increases in TRIM32 expression in podocytes following HG exposure. Functional studies showed that the inhibition of TRIM32 improved the viability of HG-stimulated podocytes, but suppressed HGinduced apoptosis, oxidative stress and the inflammatory response in podocytes. Further investigation revealed a regulatory effect of TRIM32 on Nrf2 signaling in podocytes following HG exposure. However, the suppression of Akt abrogated the TRIM32-knockdown-mediated activation of Nrf2 in HG-exposed podocytes. In addition, the knockdown of Nrf2 markedly abolished TRIM32-inhibition-induced protective effects in HG-exposed podocytes. Taken together, these results show that the inhibition of TRIM32 protects podocytes from HG-induced injury by potentiating Nrf2 signaling via modulation of the Akt/GSK$3 \beta$ axis.

\section{Materials And Methods}

Cell Culture and HG Treatment. The conditionally immortalized mouse podocyte cell line MPC5 was provided by BeNa Biotechnology (Beijing, China). The mouse podocytes were maintained in RPMI-1640 medium containing $10 \%$ fetal bovine serum (FBS) and $10 \mathrm{U} / \mathrm{ml}$ interferon- $\gamma$ at $33^{\circ} \mathrm{C}$ for cell proliferation. To induce differentiation, the medium was replaced with fresh medium without interferon- $\gamma$ and cells were cultivated at $37^{\circ} \mathrm{C}$ for 14 days. To induce the HG injury of podocytes, MPC5 cells were placed in medium containing $30 \mathrm{mM}$ glucose and cultivated for $48 \mathrm{~h}$. Cells maintained in medium containing normal glucose (NG, $5.5 \mathrm{mM}$ ) were utilized as a control.

Real-Time Quantitative PCR (RT-qPCR). Total RNAs in cultured podocytes were extracted and purified using an RNApure Tissue \& Cell Kit (Cowin Biosciences, Beijing, China). Total RNAs were reversely transcribed into cDNA using a HiFi-Script cDNA Synthesis Kit (Cowin Biosciences). FastSYBR Mixture (Cowin Biosciences) was adopted to amplify cDNA by RT-qPCR with appropriate primers. The generated 
data by RT-qPCR was assessed via $2^{-\Delta \Delta C t}$ method, and relative gene expression was obtained using GAPDH for normalization.

Western Blotting. Cultured podocytes were collected and lysed into lysis buffer (Solarbio, Beijing, China) supplemented with phosphatase inhibitors, proteinase inhibitors and phenylmethanesulfonyl fluoride. After being lysed, the lysates were centrifuged and the supernatants were collected. Protein concentration in the supernatants was quantified via a BCA Protein Assay Kit (Tiangen Biotech, Beijing, China). The same amounts of proteins were placed into each lane of sodium dodecyl sulfate (SDS) polyacrylamide gels, followed by being separated via electrophoresis. Then, proteins were transferred to Polyvinylidene Fluoride membranes via an electro-transfer method using Bio-Rad Trans-Blot apparatus (Bio-Rad Laboratories, Hercules, CA, USA). Polyvinylidene fluoride membranes were immersed into 5\% skim milk for blocking prior to incubation with primary antibodies. The membranes were hybridized with matched secondary antibody, followed by incubation with enhanced chemiluminescent reagents (Solarbio) for the visualization of protein bands. The primary antibodies against TRIM32 (Abcam, Cambridge, UK), Akt (Proteintech Group, Wuhan, China), phospho-Akt (Ser473) (Proteintech Group), GSK-3 $\beta$ (Proteintech Group), phopho-GSK-3 $\beta$ (Ser9) (Proteintech Group), Nrf2 (Proteintech Group), GAPDH (Proteintech Group) and Lamin B1 (Proteintech Group).

Cell Transfection. The siRNAs targeting TRIM32 or Nrf2 were synthesized via Genepharma (Shanghai, China). The transfection of siRNAs into podocytes was implemented via using TransIntro EL Transfection Reagent (Transgen, Beijing, China) according to the protocol provided by the manufacturer. The downregulation of target genes was confirmed by RT-qPCR or western blotting after $48 \mathrm{~h}$ transfection.

Cell Viability Assay. MPC5 podocytes were cultured in a 96-well plate and transfected with indicated siRNAs when they reached $\sim 70 \%$ confluence. After transfection for $48 \mathrm{~h}$, the medium was replaced with fresh medium harboring $\mathrm{HG}$, and podocytes were cultivated for a further $48 \mathrm{~h}$. Then, cell counting kit-8 (CCK-8) reagents (Solarbio, Beijing, China) were added to each well to determine the viability of podocytes. The absorbance of each well at $450 \mathrm{~nm}$ was measured via a microplate reader (BioTeke, Beijing, China).

Terminal Deoxynucleotidyl Transferase dUTP Nick end Labeling (TUNEL) Assay. TUNEL assay was carried out using the TransDetect In Situ Fluorescein TUNEL Cell Apoptosis Detection Kit (Transgen, Beijing, China) following the manufacturer's protocol. In brief, podocytes were fixed by formaldehyde fixing solution at the time of detection. Then, $0.1 \%$ Triton $\mathrm{X}-100$ solution was adopted to permeabilize the podocytes. Afterwards, podocytes were incubated with TdT reagent and Labeling Solution at $37^{\circ} \mathrm{C}$ for $1 \mathrm{~h}$, protected from light. After being stained with DAPI, cells were visualized via a fluorescence microscope.

Annexin V-FITC/PI Apoptosis Assay. Annexin V-FITC/PI apoptosis assay was performed via flow cytometry analysis using an Annexin V-FITC/PI Apoptosis Kit (Solarbio, Beijing, China). Briefly, cultured podocytes were dissociated by trypsin digestion and washed with ice-cold phosphate buffer saline (PBS). Podocytes were collected and re-suspended into Binding Buffer, followed by adding Annexin V-FITC/PI 
Solution. After being cultivated for $15 \mathrm{~min}$ at room temperature protected from light, cells were assessed via the FACScan flow cytometry system.

Detection of ROS Generation. The intracellular levels of ROS was evaluated via 2',7'-

dichlorodihydrofluorescein diacetate (DCFH-DA) which can be oxidized into the fluorescent DCFH.

Generally, at the time of detection, the old medium was discarded and fresh medium supplemented with $10 \mu \mathrm{M}$ DCFH-DA (Beyotime, Shanghai, China) was added to cells. After being cultivated for $30 \mathrm{~min}$ at $37^{\circ} \mathrm{C}$, cells were washed with PBS and then analyzed via the FACScan flow cytometry system to quantify the fluorescence intensity.

Measurement of Malondialdehyde (MDA) and Superoxide Dismutase (SOD) Activity. The contents of MDA and SOD in podocytes were measured by Lipid Peroxidation MDA Assay Kit (Beyotime, Shanghai, China) and Total Superoxide Dismutase Assay Kit (Beyotime), respectively, according to the protocols of the manufacturer.

Enzyme-Linked Immuno-Sorbent Assay (ELISA). The levels of pro-inflammatory cytokine levels, including interleukin (IL)-6, the tumor necrosis factor- $a$ (TNF-a), and IL-1 $\beta$ in the supernatants of cultured podocytes were quantified using ELISA kits (R\&D Systems, Minneapolis, MN, USA) following the manufacturer's protocol.

Luciferase Activity Assays. Luciferase reporter vector pARE (Beyotime, Shanghai, China), which contains an ARE binding site, was adopted to measure the transcriptional activity of Nrf2. The luciferase reporter vector pNF-KB (Beyotime) was utilized to detect the transcriptional activity of NF-KB. TRIM32 siRNAs and corresponding luciferase reporter vectors were co-transfected into MPC5 podocytes and cultivated for 48 h prior to HG stimulation. Then, cells were collected and lysed to measure luciferase activity using a Luciferase Reporter Gene Assay Kit (Beyotime).

Statistical Analysis. Experimental results were expressed as mean \pm standard deviation. Statistical analysis and graphing were implemented using GraphPad Prism 8. Student's t test was adopted for the two-group comparison. When there were three or more groups, comparisons were performed using oneway analysis of variance (ANOVA). Differences were considered statistically significant when $p<0.05$.

\section{Results}

\section{TRIM32 Expression was Elevated in Podocytes Exposed to HG}

To determine the possible relevance of TRIM32 in mediating HG-induced podocyte injury, we first analyzed the expression change of TRIM32 in MPC5 cells following exposure to HG. Our results showed that TRIM32 mRNA levels were obviously elevated in podocytes after HG treatment (Fig. 1A). Moreover, data for western blotting demonstrated significant increases in TRIM32 protein in HG-treated podocytes (Fig. 1B and C). The data indicate that TRIM32 is induced by HG in podocytes. 


\section{The Inhibition of TRIM32 Repressed HG-Induced Apoptosis of Podocytes}

To understand the biological role of TRIM32 in mediating HG-induced podocyte injury, the loss-offunction experiment of TRIM32 was carried out. Transfecting TRIM32 siRNA into MPC5 cells markedly depleted TRIM32 expression with or without HG treatment (Fig. 2A-C). The viability assay showed that HG stimulation significantly reduced the viability of podocytes, which was markedly reversed by TRIM32 knockdown (Fig. 2D). The TUNEL assay showed that HG-induced apoptosis in MPC5 cells was significantly attenuated by TRIM32 knockdown (Fig. 2E and F). Moreover, the inhibitory effect of TRIM32 knockdown on HG-induced podocyte apoptosis was further confirmed by the Annexin V-FITC/PI apoptosis assay (Fig. $2 \mathrm{G}$ and H). Collectively, these data suggest that the inhibition of TRIM32 alleviates HG-induced podocyte apoptosis.

\section{The Inhibition of TRIM32 Relieved HG-Induced Oxidative Stress in Podocytes}

To further explore the role of TRIM32 in mediating HG-induced podocyte injury, we investigated the effect of TRIM32 inhibition on HG-induced oxidative stress in MPC5 cells. HG exposure caused high generation of ROS in podocytes, which was markedly decreased by TRIM32 knockdown (Fig. 3A and B). Moreover, the HG-induced elevation in MDA contents was also reduced by TRIM32 inhibition in podocytes (Fig. 3C). In addition, SOD activity, which was inhibited by HG, was significantly increased by TRIM32 inhibition in podocytes (Fig. 3D). Altogether, these data indicate that TRIM32 inhibition relieves HG-induced oxidative stress in podocytes.

\section{The Inhibition of TRIM32 Repressed the HG-Induced Inflammatory Response in Podocytes}

We next investigated the role of TRIM32 inhibition on the HG-induced inflammatory response in podocytes. We found that the knockdown of TRIM32 prominently decreased the release of proinflammatory cytokines, including IL-6, TNF- $a$ and IL-1 $\beta$ (Fig. 4A-C). Moreover, the knockdown of TRIM32 markedly suppressed the activation of NF-KB in HG-exposed podocytes (Fig. 4D-F). Overall, these data suggest that the inhibition of TRIM32 decreases the HG-induced inflammatory response in podocytes.

\section{The Inhibition of TRIM32 Potentiated Nrf2 Signaling in HG- Exposed Podocytes}

To determine the molecular mechanism underlying TRIM32 inhibition-mediated effects in HG-exposed podocytes, we investigated the role of TRIM32 inhibition on Nrf2 signaling that contributes to the mediation of HG-induced apoptosis, oxidative stress and the inflammatory response in podocytes. The results showed that HG treatment decreased the level of nuclear Nrf2, which could be significantly up- 
regulated by TRIM32 knockdown (Fig. 5A and B). Furthermore, the knockdown of TRIM32 markedly increased the transcriptional activity of Nrf2 (Fig. 5C). In addition, the knockdown of TRIM32 enhanced the expression of Nrf2 target genes, including HO-1 and NQO-1 (Fig. 5D). In short, these data imply that the inhibition of TRIM32 potentiates Nrf2 signaling in HG-exposed podocytes.

The Inhibition of TRIM32 Enhanced Nrf2 Signaling via Modulation of the Akt/GSK-3 $\beta$ Axis in HG-Exposed Podocytes

TRIM32 plays a vital role in mediating the activation of Akt/GSK-3 $\beta$ axis. Considering that the Akt/GSK$3 \beta$ axis also regulates the activation of Nrf2 signaling, we sought to determine whether TRIM32 modulates Nrf2 signaling via the Akt/GSK-3 $\beta$ axis in HG-exposed podocytes. Interestingly, we found that TRIM32 inhibition increased the phosphorylation of Akt and GSK-3 $\beta$ in HG-exposed podocytes (Fig. 6A-C). Treatment with Akt inhibitors markedly decreased the phosphorylation of Akt and GSK-3 $\beta$ induced by TRIM32 inhibition in HG-exposed podocytes (Fig. 6A-C). Notably, TRIM32 inhibition-induced Nrf2 activation in HG-exposed podocytes was markedly abolished by Akt inhibition (Fig. 6D-F). In summary, these findings confirm that the inhibition of TRIM32 enhances Nrf2 signaling via modulation of the Akt/GSK-3ß axis.

\section{The Knockdown of Nrf2 Reversed TRIM32-Inhibition- Mediated Protective Effects in HG-Exposed Podocytes}

To confirm whether TRIM32 inhibition protects podocytes from HG injury via Nrf2 signaling, we detected the effect of Nrf2 knockdown on TRIM32-inhibition-mediated effects in HG-exposed podocytes. The transfection of Nrf2 signaling markedly decreased the activation of Nrf2 induced by TRIM32 inhibition in HG-exposed podocytes (Fig. 7A-C). As expected, the knockdown of Nrf2 significantly abolished TRIM32inhibition-induced suppressive effects on HG-induced apoptosis (Fig. 7D and E) and ROS generation (Fig. 7F and G). In addition, the inhibitory effects of TRIM32 inhibition on the HG-induced release of IL-6, TNF-a and IL-1 $\beta$ were also partially reversed by Nrf2 knockdown (Fig. $7 \mathrm{H}-\mathrm{J}$ ). To summarize, these data confirm that TRIM32 inhibition protects podocytes from HG injury via Nrf2 signaling.

\section{Discussion}

In the present work, we have determined the pivotal role of TRIM32 in regulating HG-evoked injury of podocytes. TRIM32 expression was significantly elevated in podocytes stimulated by HG. The inhibition of TRIM32 by siRNA-mediated gene silencing markedly suppressed HG-evoked podocyte apoptosis, oxidative stress and inflammatory response. Moreover, we further identified that the inhibition of TRIM32 conferred anti-HG injury in podocytes by potentiating Nrf2 signaling via regulation of the Akt/GSK-3 $\beta$ axis (Fig. 8). Overall, our work indicates a vital role of the TRIM32/Akt/GSK-3 $\beta /$ Nrf2 axis in mediating HGinduced podocyte injury, highlighting the possible relevance of TRIM32 in diabetic nephropathy. 
TRIM32 exerts a key role in regulating the survival and apoptosis of various cell types. TRIM32 can inhibit the apoptosis of cancer cells and enhances cell survival under exposure to chemotherapeutics [38-40]. The overexpression of TRIM32 protects keratinocytes from apoptosis induced by ultraviolet $b$ and tumor necrosis factor-a [23]. Despite the anti-apoptotic function of TRIM32 having been documented, the proapoptotic role of TRIM32 has been also reported in certain contexts. In TRIM32-knockout mice, traumatic brain injury-induced cell apoptosis in cortex is markedly reduced compared that in wild-type mice [41]. Moreover, the knockdown of TRIM32 alleviates oxygen-glucose deprivation-induced apoptosis of hippocampal neurons [42]. The silencing of TRIM32 decreases the apoptosis of nucleus pulposus cells induced by IL-1 $\beta$ or TNF-a [43]. Notably, TRIM32 expression is induced by hydrogen peroxide or rotenone in human embryonic kidney cells, and TRIM32 up-regulation enhances human embryonic kidney cells to hydrogen peroxide- or rotenone-induced cell death [23]. Therefore, these studies indicate that the inhibition of TRIM32 is conducive to survival under noxious stimuli. However, whether TRIM32 is involved in regulating podocyte apoptosis induced by HG is unknown. Herein we found that HG stimulation markedly up-regulated TRIM32 expression in the mouse podocyte MPC5 in vitro. Notably, the inhibition of TRIM32 improved the viability of HG-injured podocytes, and attenuated HG-induced podocyte apoptosis. Collectively, our work confirms that the inhibition of TRIM32 exerts an anti-apoptotic role in mediating HGinduced podocyte injury.

TRIM32 is involved in modulating oxidative stress. The overexpression of TRIM32 enhances the production of ROS induced by hydrogen peroxide or rotenone [23]. The inhibition of TRIM32 decreases the generation of ROS induced by oxygen-glucose deprivation in hippocampal neurons, and up-regulates the contents of SOD [42]. In line with these findings, we found that the knockdown of TRIM32 inhibited the production of ROS and MDA, while increasing the contents of SOD in podocytes exposed to HG. Therefore, our work confirms the crucial role of TRIM32 in modulating oxidative stress.

TRIM32 plays a vital role in regulating inflammatory response. TRIM32-kncok out mice have reduced production of pro-inflammatory cytokines and chemokines post $S$. suis infection [24]. The overexpression of TRIM32 promotes the release of pro-inflammatory cytokines in TNF-a-stimulated fibroblast-like synoviocytes [44]. Consistent with these studies, our work showed that the inhibition of TRIM32 markedly repressed the production of pro-inflammatory cytokines induced by HG in podocytes. Moreover, TRIM32 regulates the inflammatory response associated with the modulation of NF-kB [44]. Herein, we demonstrated that the inhibition of TRIM32 impeded the nuclear translocation of NF-KB p65, and reduced the transcriptional activity of NF-KB. Therefore, our study indicates that the inhibition of TRIM32 attenuates HG-induced inflammation in podocytes associated with the down-regulation of NF-KB activation.

Nrf2 signaling plays a vital role in modulating HG-induced apoptosis, oxidative stress and inflammation of podocytes [34-36]. Interestingly, our study reported that the inhibition of TRIM32 potentiated Nrf2 signaling in HG-exposed podocytes, which is consistent with a recent study demonstrating that TRIM32 plays a role in regulating Nrf2 activation in oxygen-glucose deprivation-induced neurons [42]. Moreover, we found that TRIM32 inhibition potentiated Nrf2 signaling via modulation of the Akt/GSK-3ß axis. 
Indeed, the Akt/GSK-3 $\beta$ axis contributes to modulation of Nrf2 activation [45-47]. In addition, TRIM32 also acts a vital regulator of the Akt/GSK-3 $\beta$ axis. It is reported that the inhibition of TRIM32 enhances plakoglobin binding to PI3K, which leads to Akt activation [48]. Furthermore, the knockout of TRIM32 significantly increased the phosphorylation of Akt and GSK-3 $\beta$ in cardiomyocytes under hypertrophic stresses [49]. Notably, our data demonstrated that the inhibition of Akt markedly reversed TRIM32inhibition-mediated Nrf2 activation in HG-exposed podocytes. Therefore, our data confirm that TRIM32 inhibition potentiates Nrf2 signaling via modulation of the Akt/GSK-3 $\beta$ axis.

\section{Conclusion}

Taken together, the findings of this study demonstrate that the inhibition of TRIM32 ameliorates HGinduced apoptosis, oxidative stress and inflammatory responses in podocytes by potentiating Nrf2

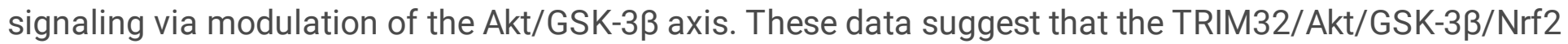
axis is a new mechanism in regulating the HG-induced injury of podocytes. This work indicates that TRIM32-mediated podocyte injury may play a vital role in the pathogenesis of diabetic nephropathy, and suggests TRIM32 as a potential target for podocyte protection. However, the precise role of TRIM32 in mediating diabetic nephropathy requires further investigation using animal models in vivo.

\section{Declarations}

\section{ETHICS APPROVAL AND CONSENT TO PARTICIPATE}

Not Applicable.

\section{CONSENT FOR PUBLICATION}

All authors have agreed for the publication of this paper.

\section{AVAILABILITY OF DATA AND MATERIALS}

The data that support the findings of this study are available on request from the corresponding author.

\section{COMPETING INTERESTS}

The authors declare that they have no conflicts of interest.

\section{FUNDING}

This study was supported by Science and Technology Research and Development Program of Shaanxi Province (2016SF-176).

\section{AUTHOR CONTRIBUTIONS}


Zhao Chen designed the study, performed the experiments and drafted the manuscript. Lifang Tian performed the experiments. Li Wang performed the experiments. Xiaotao Ma performed the experiments. Fuqian Lei collected and analyzed the data. Xianghui Chen collected and analyzed the data. Rongguo Fu designed the study and reviewed the manuscript. All authors have read and approved the final version of this manuscript.

\section{ACKNOWLEDGEMENTS}

Not Applicable.

\section{References}

1. Gnudi L., R.J.M. Coward, D.A. Long. 2016. Diabetic nephropathy: perspective on novel molecular mechanisms. Trends Endocrinol Metab 27: 820-830.

2. Wada J., H. Makino. 2013. Inflammation and the pathogenesis of diabetic nephropathy. Clin Sci (Lond) 124: 139-152.

3. Gheith O., N. Farouk, N. Nampoory, M.A. Halim, T. Al-Otaibi. 2016. Diabetic kidney disease: world wide difference of prevalence and risk factors. J Nephropharmacol 5: 49-56.

4. Armelloni S., A. Corbelli, L. Giardino, M. Li, M. Ikehata, D. Mattinzoli, P. Messa, C. Pignatari, S. Watanabe, M.P. Rastaldi. 2014. Podocytes: recent biomolecular developments. Biomol Concepts 5: 319-330.

5. Mitjavila M.T., J.J. Moreno. 2012. The effects of polyphenols on oxidative stress and the arachidonic acid cascade. Implications for the prevention/treatment of high prevalence diseases. Biochem Pharmacol 84: 1113-1122.

6. Elmarakby A.A., J.C. Sullivan. 2012. Relationship between oxidative stress and inflammatory cytokines in diabetic nephropathy. Cardiovasc Ther 30: 49-59.

7. Ram C., A.K. Jha, A. Ghosh, S. Gairola, A.M. Syed, U.S. Murty, V.G.M. Naidu, B.D. Sahu. 2020. Targeting NLRP3 inflammasome as a promising approach for treatment of diabetic nephropathy: Preclinical evidences with therapeutic approaches. Eur J Pharmacol 885: 173503.

8. Watanabe M., S. Hatakeyama. 2017. TRIM proteins and diseases. J Biochem 161: 135-144.

9. Cohen S., B. Zhai, S.P. Gygi, A.L. Goldberg. 2012. Ubiquitylation by Trim32 causes coupled loss of desmin, Z-bands, and thin filaments in muscle atrophy. J Cell Biol 198: 575-589.

10. Izumi H., Y. Kaneko. 2014. Trim32 facilitates degradation of MYCN on spindle poles and induces asymmetric cell division in human neuroblastoma cells. Cancer Res 74: 5620-5630.

11. Kano S., N. Miyajima, S. Fukuda, S. Hatakeyama. 2008. Tripartite motif protein 32 facilitates cell growth and migration via degradation of Abl-interactor 2. Cancer Res 68: 5572-5580.

12. Shieh P.B., E. Kudryashova, M.J. Spencer. 2011. Limb-girdle muscular dystrophy $2 \mathrm{H}$ and the role of TRIM32. Handb Clin Neurol 101: 125-133. 
13. Servian-Morilla E., M. Cabrera-Serrano, E. Rivas-Infante, A. Carvajal, P.J. Lamont, A.L. Pelayo-Negro, G. Ravenscroft, R. Junckerstorff, J.M. Dyke, S. Fletcher, et al. 2019. Altered myogenesis and premature senescence underlie human TRIM32-related myopathy. Acta Neuropathol Commun 7: 30.

14. Panicucci C., M. Traverso, S. Baratto, C. Romeo, M. Iacomino, C. Gemelli, A. Tagliafico, P. Broda, F. Zara, C. Bruno, et al. 2019. Novel TRIM32 mutation in sarcotubular myopathy. Acta Myo/38: 8-12.

15. Borlepawar A., A.Y. Rangrez, A. Bernt, L. Christen, S. Sossalla, D. Frank, N. Frey. 2017. TRIM24 protein promotes and TRIM32 protein inhibits cardiomyocyte hypertrophy via regulation of dysbindin protein levels. J Biol Chem 292: 10180-10196.

16. Ntim M., Q.F. Li, Y. Zhang, X.D. Liu, N. Li, H.L. Sun, X. Zhang, B. Khan, B. Wang, Q. Wu, et al. 2020. TRIM32 deficiency impairs synaptic plasticity by excitatory-inhibitory imbalance via Notch pathway. Cereb Cortex 30: 4617-4632.

17. Hillje A.L., E. Beckmann, M.A. Pavlou, C. Jaeger, M.P. Pacheco, T. Sauter, J.C. Schwamborn, L. Lewejohann. 2015. The neural stem cell fate determinant TRIM32 regulates complex behavioral traits. Front Cell Neurosci 9: 75.

18. Ruan C.S., S.F. Wang, Y.J. Shen, Y. Guo, C.R. Yang, F.H. Zhou, L.T. Tan, L. Zhou, J.J. Liu, W.Y. Wang, et al. 2014. Deletion of TRIM32 protects mice from anxiety- and depression-like behaviors under mild stress. Eur J Neurosci 40: 2680-2690.

19. Yin H., Z. Li, J. Chen, X. Hu. 2019. Expression and the potential functions of TRIM32 in lung cancer tumorigenesis. J Cell Biochem 120: 5232-5243.

20. Wang M., W. Luo, Y. Zhang, R. Yang, X. Li, Y. Guo, C. Zhang, R. Yang, W.Q. Gao. 2020. Trim32 suppresses cerebellar development and tumorigenesis by degrading Gli1/sonic hedgehog signaling. Cell Death Differ 27: 1286-1299.

21. Zhu J.W., M.M. Zou, Y.F. Li, W.J. Chen, J.C. Liu, H. Chen, L.P. Fang, Y. Zhang, Z.T. Wang, J.B. Chen, et al. 2020. Absence of TRIM32 leads to reduced GABAergic interneuron generation and autism-like behaviors in mice via suppressing mTOR signaling. Cereb Cortex 30: 3240-3258.

22. Wang C., J. Xu, H. Fu, Y. Zhang, X. Zhang, D. Yang, Z. Zhu, Z. Wei, Z. Hu, R. Yan, et al. 2018. TRIM32 promotes cell proliferation and invasion by activating beta-catenin signalling in gastric cancer. $J$ Cell Mol Med 22: 5020-5028.

23. Prajapati P., D. Gohel, A. Shinde, M. Roy, K. Singh, R. Singh. 2020. TRIM32 regulates mitochondrial mediated ROS levels and sensitizes the oxidative stress induced cell death. Cell Signal 76: 109777.

24. OuYang X., J. Guo, Q. Lv, H. Jiang, Y. Zheng, P. Liu, T. Zhao, D. Kong, H. Hao, Y. Jiang. 2020. TRIM32 drives pathogenesis in streptococcal toxic shock-like syndrome and streptococcus suis meningitis by regulating innate immune responses. Infect Immun 88: e00957-00919.

25. Vomund S., A. Schafer, M.J. Parnham, B. Brune, A. von Knethen. 2017. Nrf2, the master regulator of anti-oxidative responses. Int J Mol Sci 18: 2772.

26. Zhang D.D., M. Hannink. 2003. Distinct cysteine residues in Keap1 are required for Keap1-dependent ubiquitination of Nrf2 and for stabilization of Nrf2 by chemopreventive agents and oxidative stress. Mol Cell Biol 23: 8137-8151. 
27. Gum S.I., M.K. Cho. 2013. Recent updates on acetaminophen hepatotoxicity: the role of nrf2 in hepatoprotection. Toxicol Res 29: 165-172.

28. Ishii Y., K. Itoh, Y. Morishima, T. Kimura, T. Kiwamoto, T. lizuka, A.E. Hegab, T. Hosoya, A. Nomura, T. Sakamoto, et al. 2005. Transcription factor Nrf2 plays a pivotal role in protection against elastaseinduced pulmonary inflammation and emphysema. J Immunol 175: 6968-6975.

29. Kobayashi E.H., T. Suzuki, R. Funayama, T. Nagashima, M. Hayashi, H. Sekine, N. Tanaka, T. Moriguchi, H. Motohashi, K. Nakayama, et al. 2016. Nrf2 suppresses macrophage inflammatory response by blocking proinflammatory cytokine transcription. Nat Commun 7: 11624.

30. Anedda A., E. Lopez-Bernardo, B. Acosta-Iborra, M. Saadeh Suleiman, M.O. Landazuri, S. Cadenas. 2013. The transcription factor Nrf2 promotes survival by enhancing the expression of uncoupling protein 3 under conditions of oxidative stress. Free Radic Biol Med 61: 395-407.

31. Adelusi T.I., L. Du, M. Hao, X. Zhou, Q. Xuan, C. Apu, Y. Sun, Q. Lu, X. Yin. 2020. Keap1/Nrf2/ARE signaling unfolds therapeutic targets for redox imbalanced-mediated diseases and diabetic nephropathy. Biomed Pharmacother 123: 109732.

32. Warren A.M., S.T. Knudsen, M.E. Cooper. 2019. Diabetic nephropathy: an insight into molecular mechanisms and emerging therapies. Expert Opin Ther Targets 23: 579-591.

33. Keri K.C., N.S. Samji, S. Blumenthal. 2018. Diabetic nephropathy: newer therapeutic perspectives. $J$ Community Hosp Intern Med Perspect 8: 200-207.

34. Chang T.T., Y.A. Chen, S.Y. Li, J.W. Chen. 2020. Nrf-2 mediated heme oxygenase-1 activation contributes to the anti-inflammatory and renal protective effects of Ginkgo biloba extract in diabetic nephropathy. J Ethnopharmacol 266: 113474.

35. Wang D., M. Jin, X. Zhao, T. Zhao, W. Lin, Z. He, M. Fan, W. Jin, J. Zhou, L. Jin, et al. 2019. FGF1(DeltaHBS) ameliorates chronic kidney disease via PI3K/AKT mediated suppression of oxidative stress and inflammation. Cell Death Dis 10: 464.

36. Yang S.M., S.M. Ka, H.L. Wu, Y.C. Yeh, C.H. Kuo, K.F. Hua, G.Y. Shi, Y.J. Hung, F.C. Hsiao, S.S. Yang, et al. 2014. Thrombomodulin domain 1 ameliorates diabetic nephropathy in mice via anti-NFkappaB/NLRP3 inflammasome-mediated inflammation, enhancement of NRF2 antioxidant activity and inhibition of apoptosis. Diabetologia 57: 424-434.

37. Shaw P., A. Chattopadhyay. 2020. Nrf2-ARE signaling in cellular protection: Mechanism of action and the regulatory mechanisms. J Cell Physio/ 235: 3119-3130.

38. Horn E.J., A. Albor, Y. Liu, S. El-Hizawi, G.E. Vanderbeek, M. Babcock, G.T. Bowden, H. Hennings, G. Lozano, W.C. Weinberg, et al. 2004. RING protein Trim32 associated with skin carcinogenesis has anti-apoptotic and E3-ubiquitin ligase properties. Carcinogenesis 25: 157-167.

39. Su X., B. Wang, Y. Wang, B. Wang. 2020. Inhibition of TRIM32 Induced by miR-519d Increases the Sensitivity of Colorectal Cancer Cells to Cisplatin. Onco Targets Ther 13: 277-289.

40. Du Y., W. Zhang, B. Du, S. Zang, X. Wang, X. Mao, Z. Hu. 2018. TRIM32 overexpression improves chemoresistance through regulation of mitochondrial function in non-small-cell lung cancers. Onco Targets Ther 11: 7841-7852. 
41. Zhang Z.B., L.L. Xiong, B.T. Lu, H.X. Zhang, P. Zhang, T.H. Wang. 2017. Suppression of Trim32 Enhances Motor Function Repair after Traumatic Brain Injury Associated with Antiapoptosis. Cell Transplant 26: 1276-1285.

42. Wei L., J.S. Zhang, S.F. Ji, H. Xu, Z.H. Zhao, L. Zhang, L. Pang, J.F. Zhang, P.B. Yang, H. Ma. 2019. Knockdown of TRIM32 protects hippocampal neurons from oxygen-glucose deprivation-induced injury. Neurochem Res 44: 2182-2189.

43. Chen F., Q. Guo, Q. Chen, Z. Han, X. Zhou, L. Wu, X. Guo, B. Ni, J. Yang. 2020. TRIM32 triggers betacatenin signaling through ubiquitylation of AXIN1 to promote inflammatory factor-induced apoptosis of rat nucleus pulposus cells. Am J Physiol Cell Physio/318: C695-C703.

44. Liang T., M. Song, K. Xu, C. Guo, H. Xu, H. Zhang, L. Xu. 2020. TRIM32 promotes inflammatory responses in rheumatoid arthritis fibroblast-like synoviocytes. Scand J Immuno/ 91: e12876.

45. Yu H., J. Zhen, Y. Yang, J. Du, J. Leng, Q. Tong. 2020. Rg1 protects H9C2 cells from high glucose-/palmitate-induced injury via activation of AKT/GSK-3beta/Nrf2 pathway. J Cell Mol Med 24: 8194-8205.

46. Chen R., Y.Y. Zhang, J.N. Lan, H.M. Liu, W. Li, Y. Wu, Y. Leng, L.H. Tang, J.B. Hou, Q. Sun, et al. 2020. Ischemic postconditioning alleviates intestinal ischemia-reperfusion injury by enhancing autophagy and suppressing oxidative stress through the Akt/GSK-3beta/Nrf2 pathway in mice. Oxid Med Cell Longev 2020: 6954764.

47. Li X., Y. Zou, J. Xing, Y.Y. Fu, K.Y. Wang, P.Z. Wan, X.Y. Zhai. 2020. Pretreatment with roxadustat (FG4592) attenuates folic acid-induced kidney injury through antiferroptosis via Akt/GSK-3beta/Nrf2 pathway. Oxid Med Cell Longev 2020: 6286984.

48. Cohen S., D. Lee, B. Zhai, S.P. Gygi, A.L. Goldberg. 2014. Trim32 reduces PI3K-Akt-FoxO signaling in muscle atrophy by promoting plakoglobin-PI3K dissociation. J Cell Biol 204: 747-758.

49. Chen L., J. Huang, Y. Ji, X. Zhang, P. Wang, K. Deng, X. Jiang, G. Ma, H. Li. 2016. Tripartite motif 32 prevents pathological cardiac hypertrophy. Clin Sci (Lond) 130: 813-828.

\section{Figures}

A

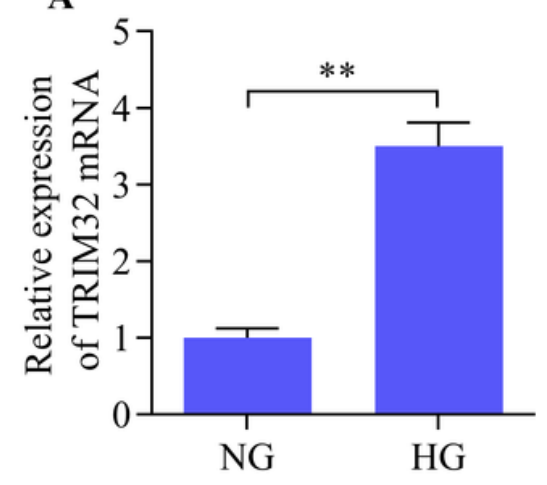

B

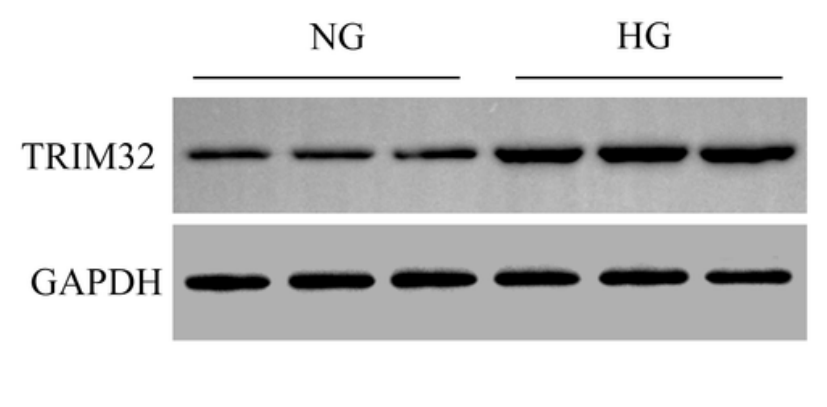

C

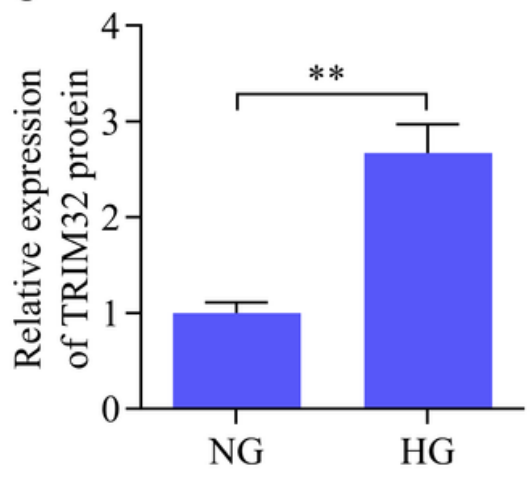

Figure 1 
The effect of HG treatment on TRIM32 expression in MPC5 cells. MPC5 cells were cultured for $48 \mathrm{~h}$ in the presence of NG or HG. (A) RT-qPCR was utilized to determine the effect of HG on TRIM32 mRNA levels. (B, C) Western blotting was adopted to measure the effect of HG on TRIM32 protein levels. $N=3,{ }^{*} p<0.01$.
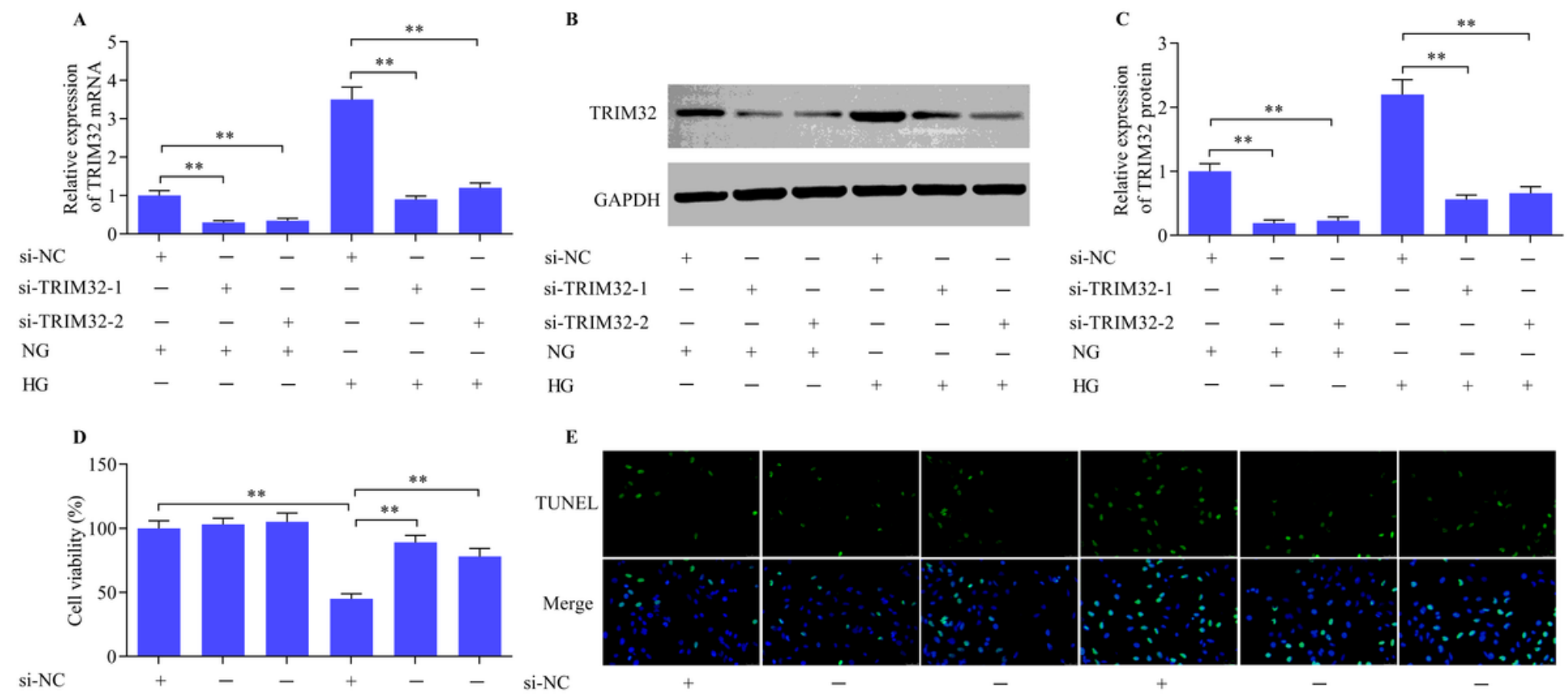

si-TRIM32-1 - + + -

- si-TRIM32-1 -

$\begin{array}{lllllllll}\text { si-TRIM32-2 } & - & - & + & - & - & + & \text { si-TRIM32-2 } & - \\ \mathrm{NG} & + & + & + & - & - & - & \mathrm{NG} & +\end{array}$

HG
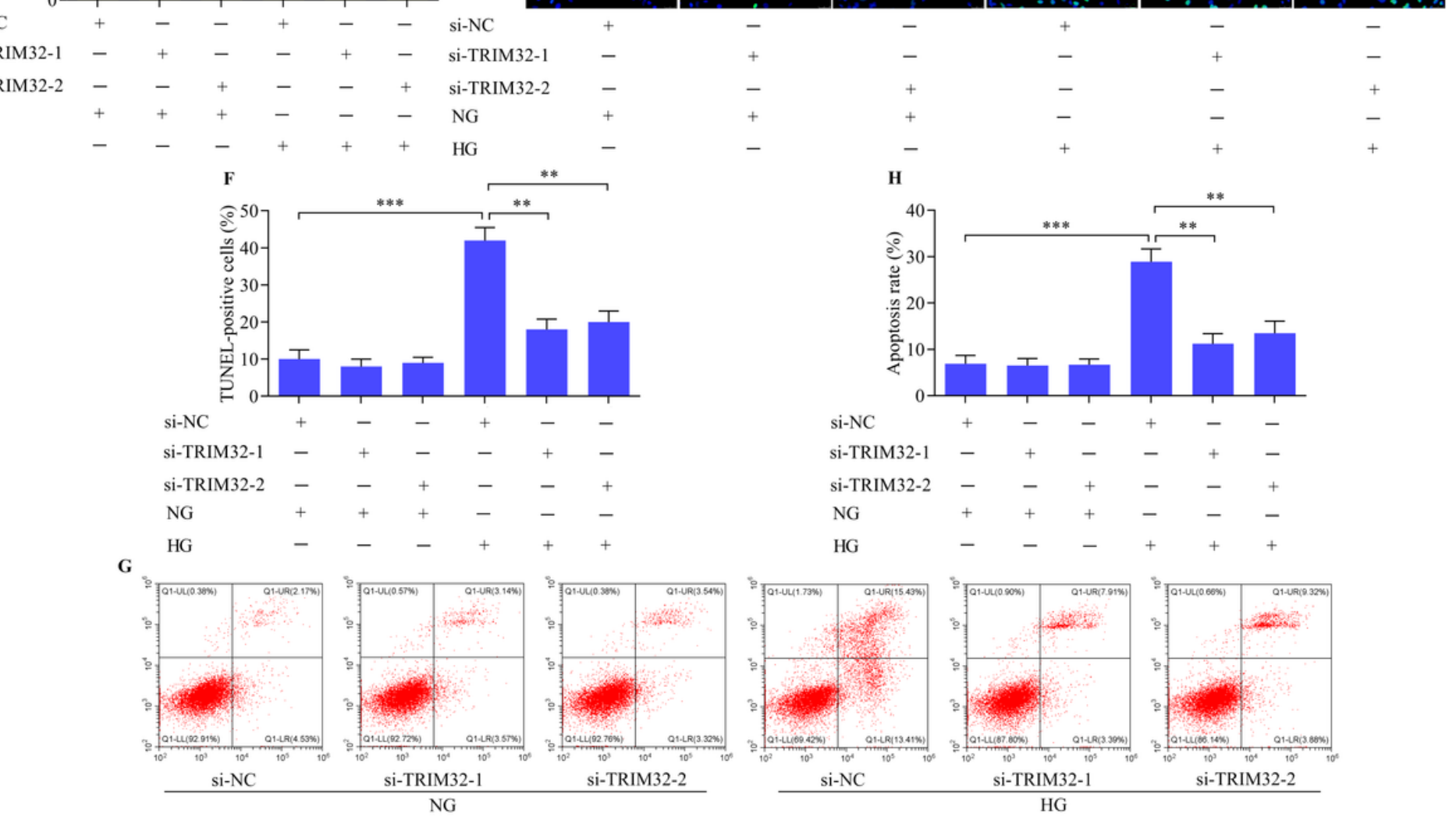

\section{Figure 2}

The effect of TRIM32 inhibition on HG-induced podocyte apoptosis. MPC5 cells were transfected with TRIM32 siRNA or control siRNA for $48 \mathrm{~h}$ and then subjected to HG exposure. The down-regulation of TRIM32 level by siRNA transfection was confirmed by (A) RT-qPCR and $(B, C)$ western blotting $(N=3)$. (D) The effect of TRIM32 inhibition on podocyte viability was assessed via CCK-8 assay ( $N=4)$. The effect of 
TRIM32 inhibition on podocyte apoptosis was evaluated via (E, F) TUNEL assay and (G, H) Annexin V$\mathrm{FITC} / \mathrm{PI}$ assay $(\mathrm{N}=3)$. ${ }^{* *} \mathrm{p}<0.01$.

A
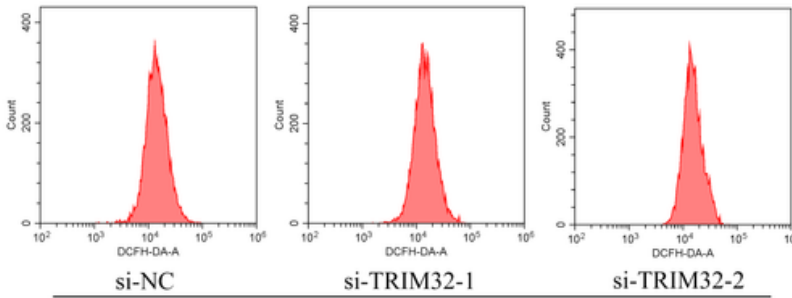

Si-TRIM32-1 si-TRIM32-2
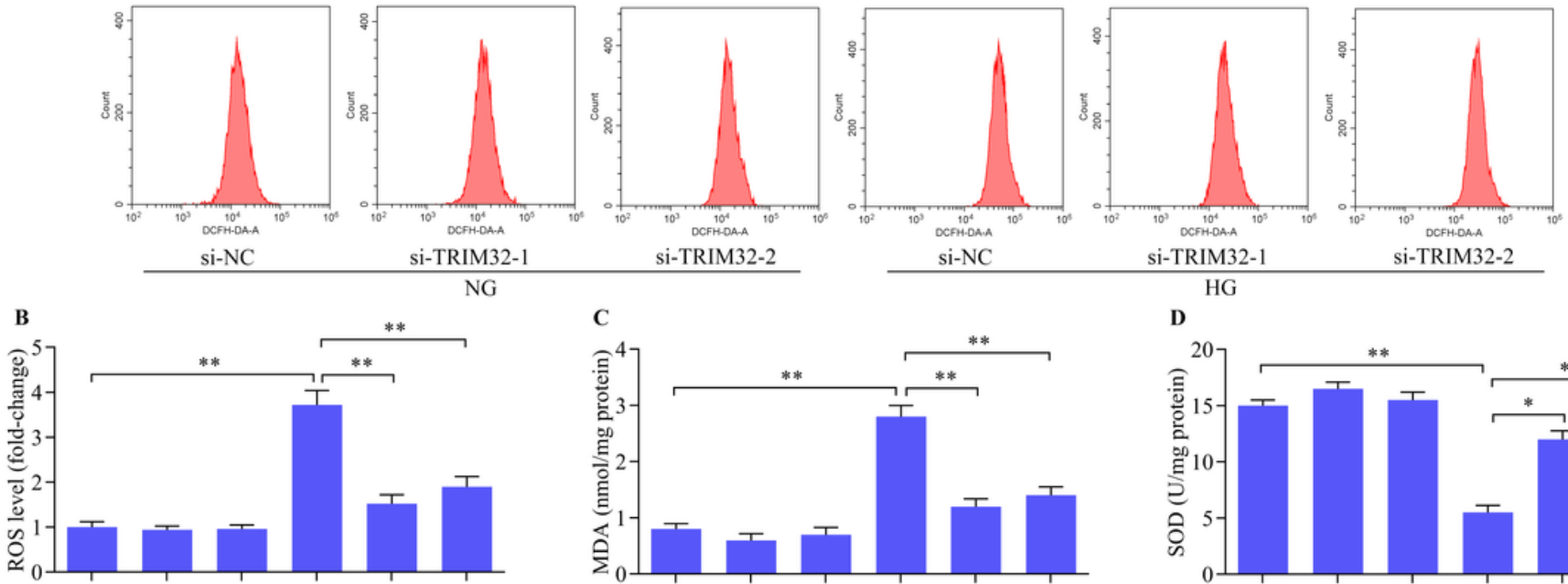

si-TRIM32-

si-TRIM32-2
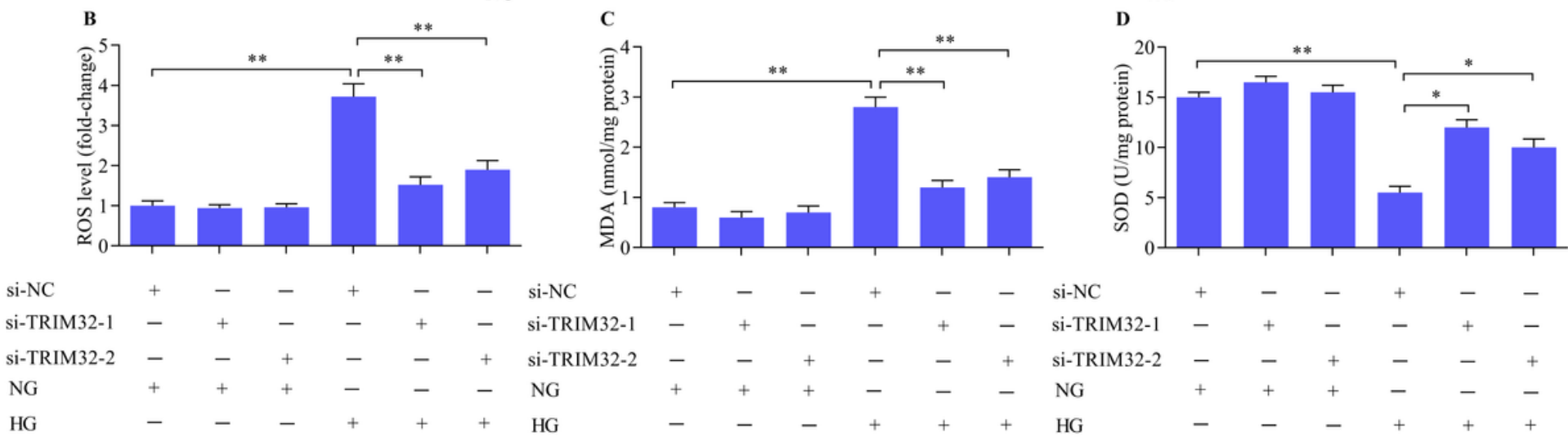

\section{Figure 3}

The effect of TRIM32 knockdown on HG-induced oxidative stress in podocytes. (A, B) The effect of TRIM32 inhibition on ROS generation was monitored via DCFH-DA staining method ( $N=3)$. (C) The effect of TRIM32 inhibition on MDA contents was assessed via MDA assay ( $N=4)$. (D) The effect of TRIM32 inhibition on SOD activity was evaluated via SOD assay $(\mathrm{N}=4)$. ${ }^{*} \mathrm{p}<0.05$ and ${ }^{* *} \mathrm{p}<0.01$.

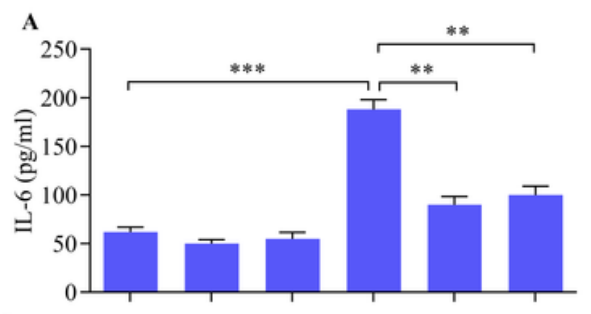

si-NC

si-TRIM32-2 $-\quad-\quad+\quad-\quad-\quad+$ si-TRIM32-2

NG

$\mathrm{HG}$

D

$\mathrm{NF}-\kappa \mathrm{B}$ p 65

Lamin B1

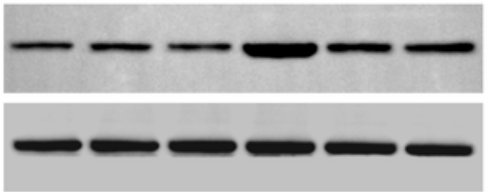

si-NC

Si-TRIM32-2

NG

HG

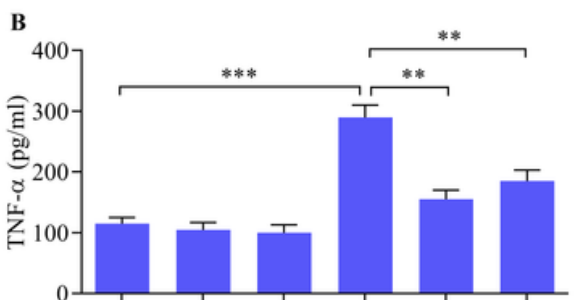

NG

$\mathrm{HG}$

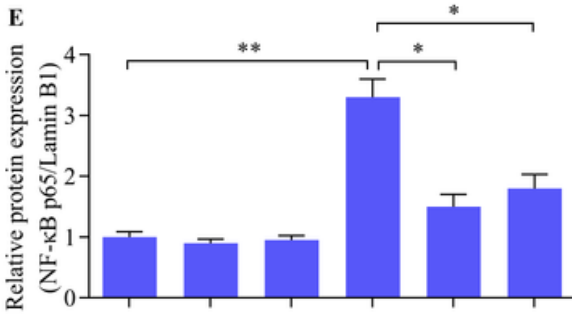

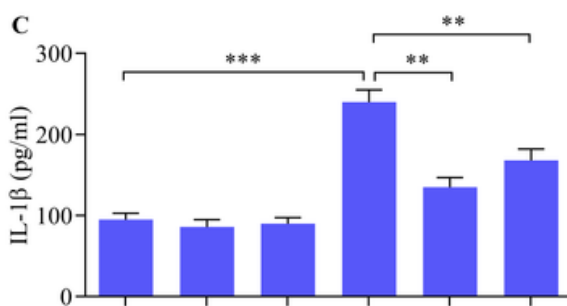

si-NC

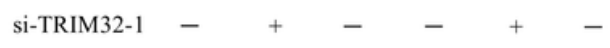

si-TRIM32-2 $-\quad-\quad+\quad-\quad-$

NG

HG

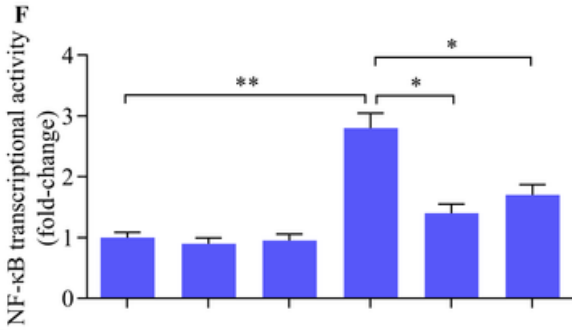

si-NC

si-TRIM32-1

si-TRIM32-2

NG

HG 


\section{Figure 4}

The effect of TRIM32 knockdown on the HG-induced inflammatory response in podocytes. The effect of TRIM32 inhibition on concentration levels of (A) IL-6, (B) TNF- $a$ and (C) IL-1 $\beta$ in the culture supernatants was measured via ELISA (N=4). (D, E) The effect of TRIM32 inhibition on the levels of nuclear NF-KB p65 protein was determined via western blotting. Lamin B1 served as the loading control $(N=3)$. (F) The effect of TRIM32 inhibition on the transcriptional activity of NF-KB was measured via luciferase reporter assay $(\mathrm{N}=5) .{ }^{*} \mathrm{p}<0.05,{ }^{* *} \mathrm{p}<0.01$ and ${ }^{* * *} \mathrm{p}<0.001$.

A

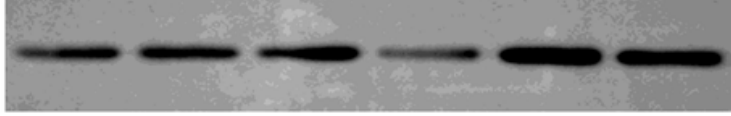

Lamin B1

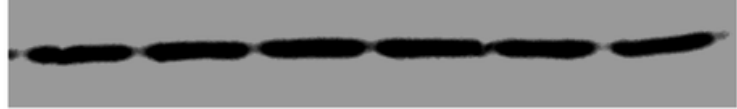

$\begin{array}{lllllll}\text { si-NC } & + & - & - & + & - & - \\ \text { si-TRIM32-1 } & - & + & - & - & + & - \\ \text { si-TRIM32-2 } & - & - & + & - & - & + \\ \text { NG } & + & + & + & - & - & - \\ \text { HG } & - & - & - & + & + & +\end{array}$

C

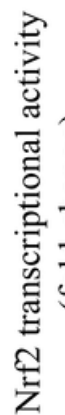



si-NC

si-TRIM32-1

si-TRIM32-2

NG

HG

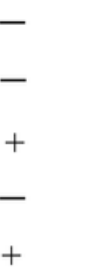

si-NC si-TRIM32-1 si-TRIM32-2 NG HG

D

HO- 1

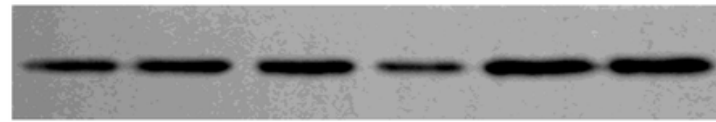

NQO-

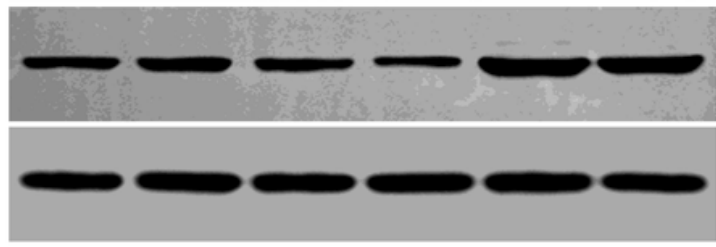

GAPDH si-NC si-TRIM32-1 si-TRIM32-2 NG HG

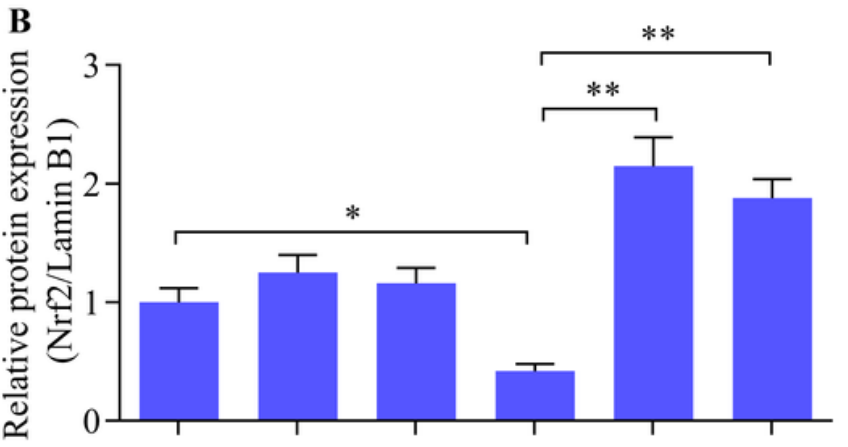

$\begin{array}{lllllll}+ & - & - & + & - & & -\end{array}$ $+\quad-\quad-\quad+\quad-$

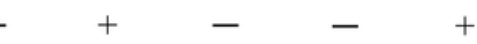
$+\quad--$ $-\quad+\quad+$ 


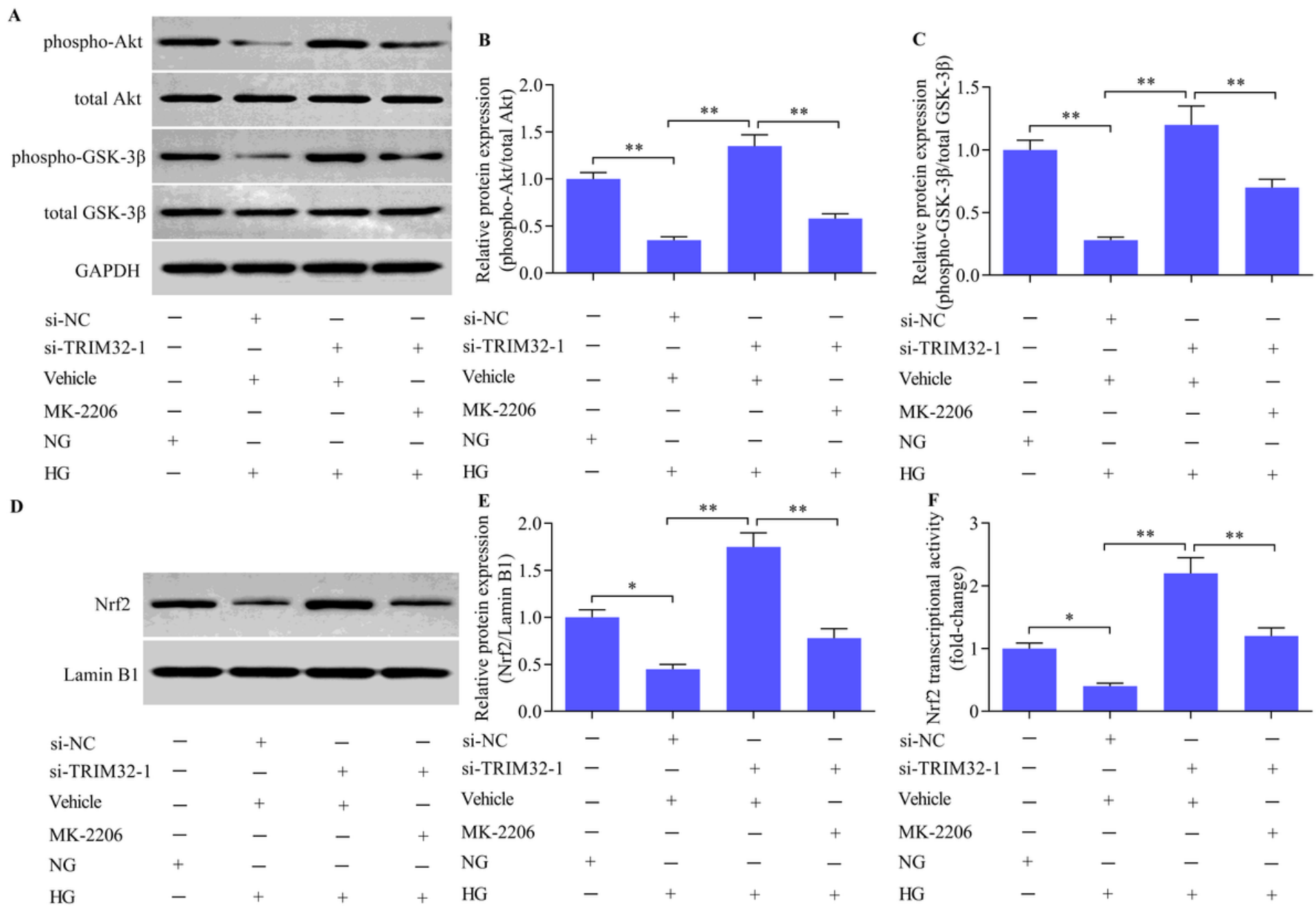

\section{Figure 6}

The effect of Akt inhibition on TRIM32-mediated Nrf2 signaling. MPC5 cells were transfected with TRIM32 siRNA for $48 \mathrm{~h}$ in the presence or absence of Akt inhibitor, MK-2206 $2 \mathrm{HCl}$, prior to HG treatment. Levels of (A-C) phospho-Akt and phospho-GSK-3 $\beta$, and (D, E) nuclear Nrf2 were determined via western blotting (N=3). (F) Nrf2 transcriptional activity was monitored via luciferase activity $(\mathrm{N}=5)$. ${ }^{*} \mathrm{p}<0.05$ and $\star * \mathrm{p}<0.01$. 
A
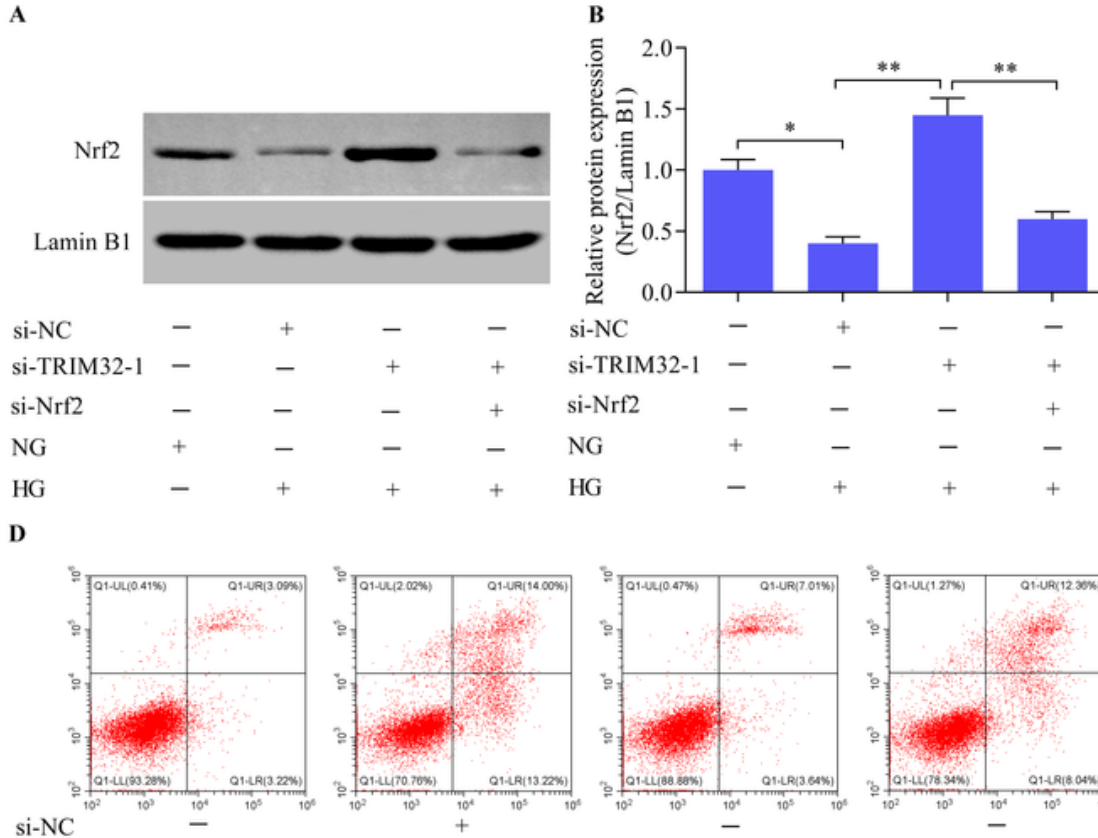

si-TRIM32-1 -

si-Nrf2

NG

$\mathrm{HG}$

F
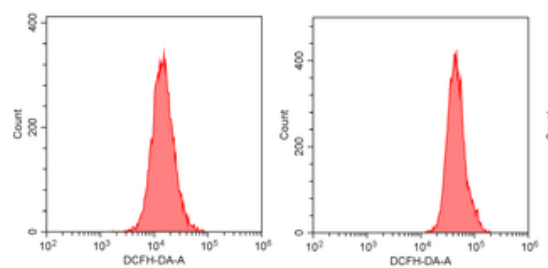

si-NC

si-TRIM32-1 -

si-Nrf2

NG

HG

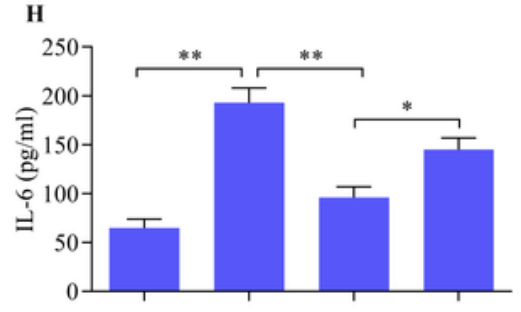

si-NC

si-TRIM32-1 -

si-Nrf2

NG

HG
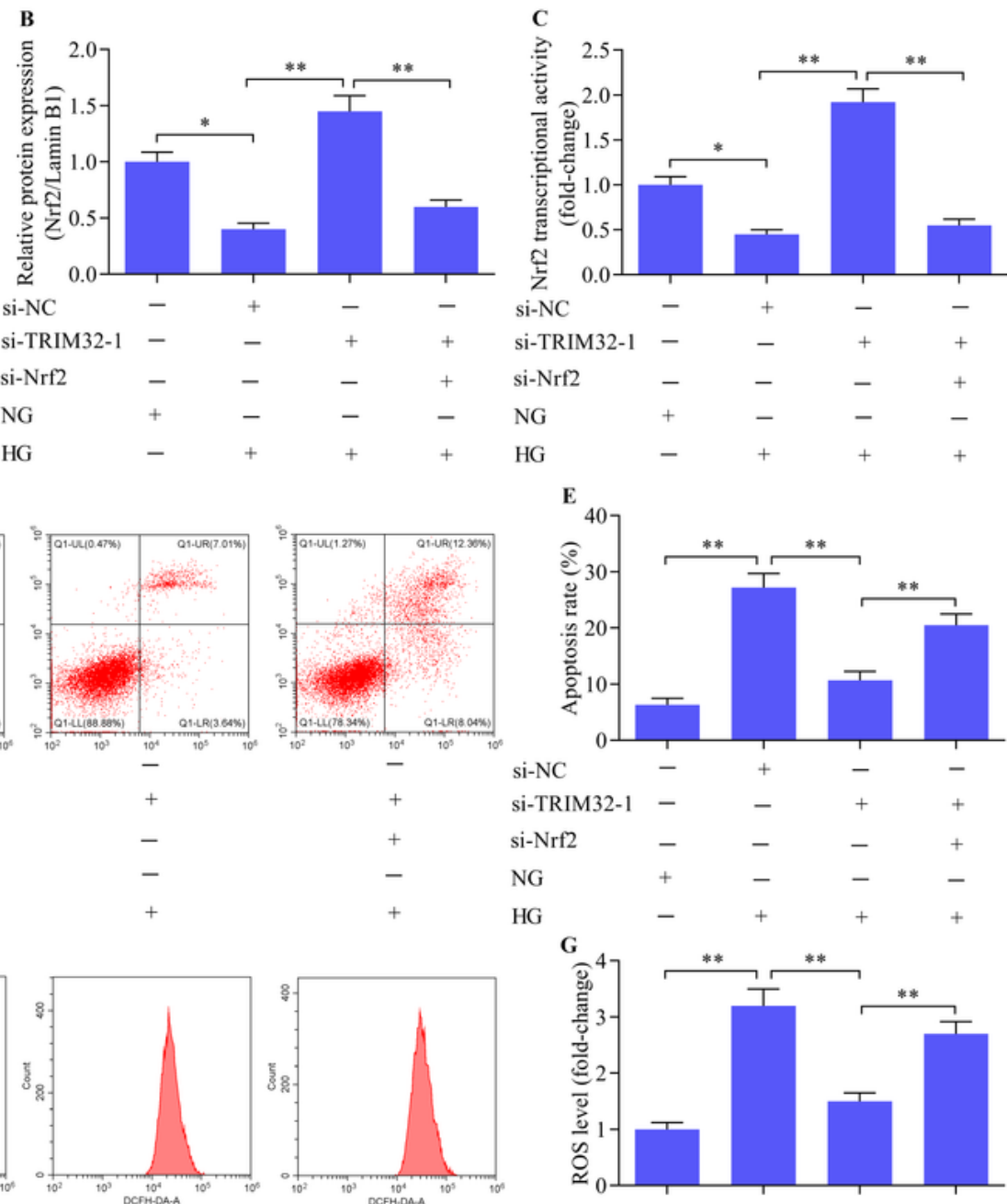

HG
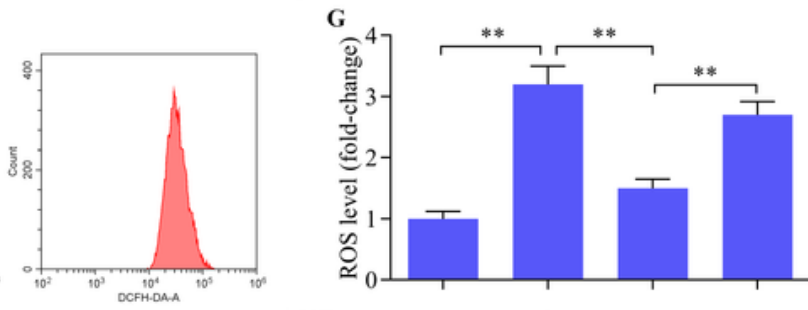

si-NC

si-TRIM32-1 -

si-Nrf2

NG

HG
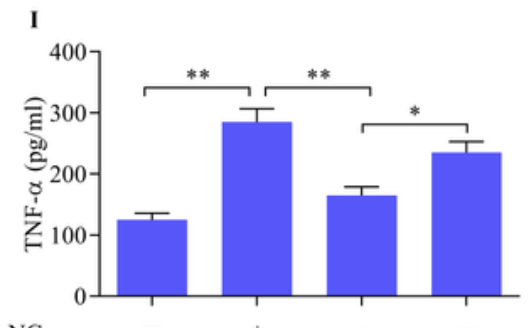

si-NC si-TRIM32-1 si-Nrf2

NG

HG
J

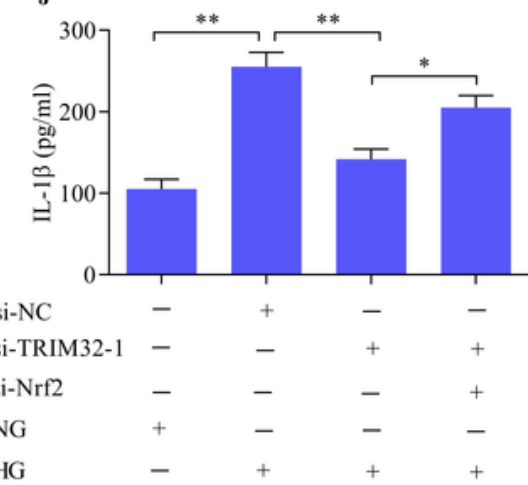

\section{Figure 7}

The effect of Nrf2 inhibition on TRIM32-inhibition-mediated protective effects in HG-exposed podocytes. TRIM32 siRNA and Nrf2 siRNA were co-transfected into MPC5 cells followed by incubation for $48 \mathrm{~h}$ before HG exposure. (A-C) Levels of nuclear Nrf2 were examined via western blotting (N=3). (D, E) Podocytes apoptosis was measured via the Annexin V-FITC/PI assay $(\mathrm{N}=3) .(\mathrm{F}, \mathrm{G})$ ROS levels in podocytes were assessed via the DCFH-DA staining method (N=3). Concentration levels of $(H) I L-6,(I)$ 
TNF- $a$ and $(J)$ IL-1 $\beta$ in culture supernatants of podocytes were monitored via ELISA $(N=4) .{ }^{*} p<0.05$ and $\star \star p<0.01$.

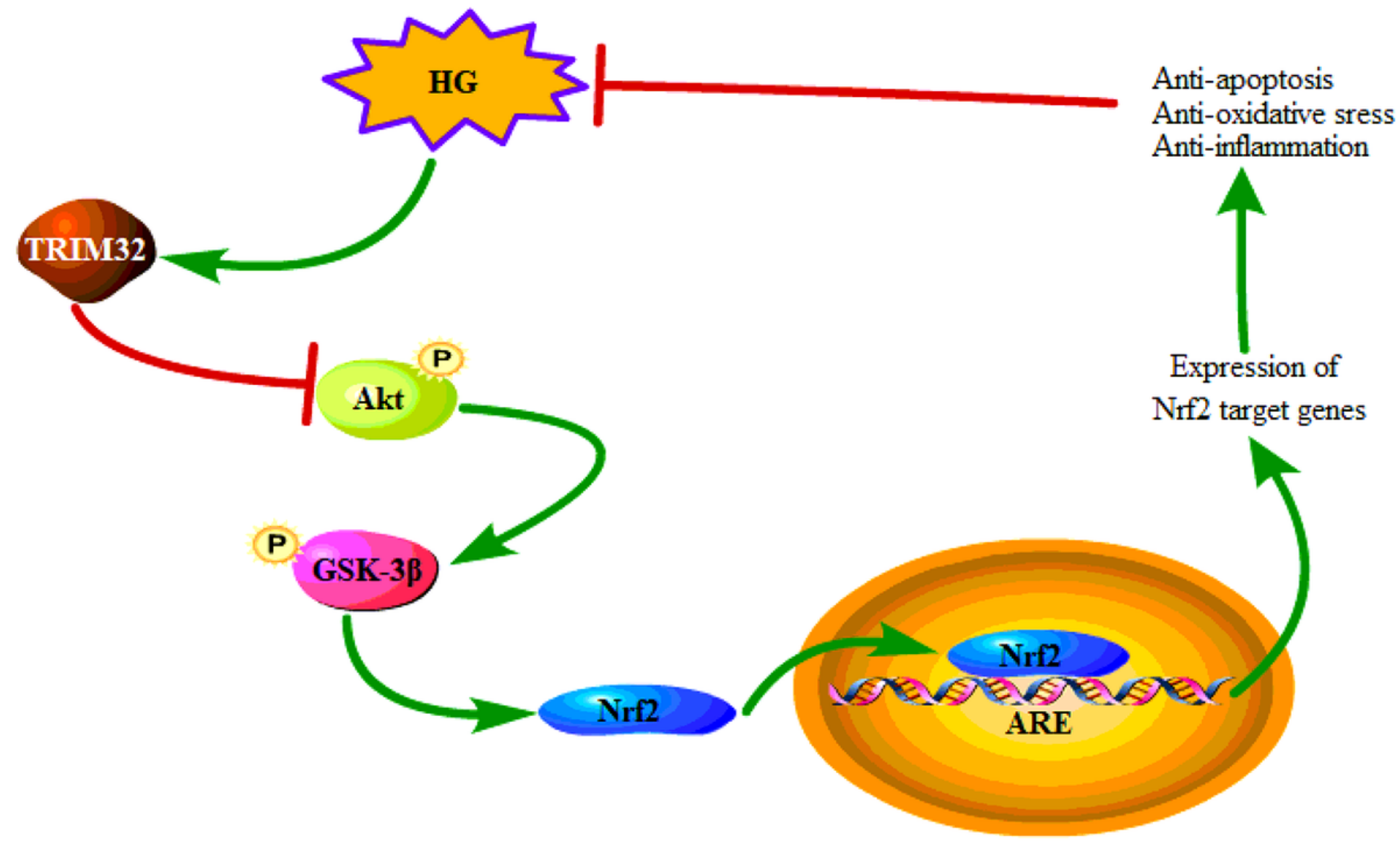

Figure 8

A proposed model for TRIM32 in mediating HG-induced podocyte injury. 